Eine neue Rolle für verantwortliche Unternehmen

\title{
Förderung des nachhaltigen Konsums von Mitarbeitern
}

\begin{abstract}
Unternehmen spielen eine zentrale Rolle nicht nur für eine nachhaltige Entwicklung insgesamt, sondern insbesondere für einen nachhaltigen Konsum. Allerdings nehmen Unternehmen die Konsumenten bisher fast ausschließlich als Kunden wahr. Dass auch die Mitarbeiter im eigenen Betrieb Konsumenten sind, wird dagegen zumeist übersehen. Von Viola Muster und Ulf Schrader
\end{abstract}

\begin{abstract}
m aktuellen Förderprogramm des Bundesministeriums für Bildung und Forschung „Neue Wege zum Nachhaltigen Konsum “ wird in fast allen Projekten intensiv mit Unternehmenspartnern zusammengearbeitet. Dabei fällt jedoch auf, dass ausschließlich Kunden, nicht aber die Mitarbeiter der Unternehmen als Konsumenten gesehen werden. Wir wollen im Folgenden zeigen, dass eine solche Sichtweise verkürzt ist. Sie wird einem ganzheitlichen Verständnis von Corporate Social Responsibility (CSR) nicht gerecht und vernachlässigt Potenziale für einen gesellschaftlichen Wandel.
\end{abstract}

\section{Ein nachhaltiges Konsum- verhalten unterstützen}

Bisher wird das private Konsumverhalten von Mitarbeitern nur zufällig beeinflusst. Maßnahmen betrieblicher Nachhaltigkeitsbildung wie Anleitungen zur Mülltrennung im Unternehmen haben dabei das Potenzial, im privaten Alltag der Mitarbeiter weiter zu wirken (Berger/Kanetkar 1995; Thøgersen 1999). Diese sogenannten Spillover-Effekte sind vor allem dann wahrscheinlich, wenn das Gelernte einerseits eine Bedeutung für das Privatleben besitzt und andererseits unproblematisch übertragen werden kann.

Doch im Hinblick auf umfangreiche Verhaltensänderungen, die zur Umsetzung nachhaltiger Konsummuster in der Gesellschaft notwendig sind, fällt die Wirkungsbilanz dieser Spillover-Effekte eher bescheiden aus. Letztlich sind sie nur ein zufälliges Nebenprodukt der betrieblichen Nachhaltigkeitsbildung. Demgegenüber liegen die unternehmerischen Handlungspotenziale, direkt und bewusst auf das private Kauf- und Verbrauchsverhalten der Mitarbeiter einzuwirken, weitgehend brach. Zum einen weil davon auszugehen ist, dass sich Unternehmen für private Entscheidungen der Mitarbeiter nicht zuständig fühlen, zum anderen, weil es moralische und rechtliche Vorbehalte gibt, auf die Privatsphäre der Mitarbeiter Einfluss zu nehmen.

Doch private Dispositionen finden in Unternehmen längst Berücksichtigung. Die Privatsphäre der Mitarbeiter ist beispielsweise unternehmensrelevant, wenn es um Flexibilität oder Mobilität geht. Auch im Hinblick auf die Kompetenzentwicklung, Reproduktionsleistungen und sogenannte „soft skills“ wie Motivation und Durchhaltevermögen haben private Interessen und Lebenslagen eines Beschäftigten längst große Bedeutung für den Arbeitgeber und können sich für die Mitarbeiter in unterschiedlichen Karrierechancen oder Gehaltsstufen niederschlagen (Jürgens 2006). Nun wird dieses Interesse an der privaten Lebensgestaltung gern damit legitimiert, dass jene Bereiche von Relevanz für das Unternehmen sind und in einer Grauzone zwischen Privatleben und Arbeit liegen.

Es erscheint also opportun, eine Unterscheidung zwischen betriebsrelevantem Privatleben und privatem Privatleben vorzunehmen und darauf basie- rend die unternehmerischen Zuständigkeiten zu rechtfertigen. In der Konsequenz kann jedoch kaum glaubhaft vermittelt werden, dass private Konsumgewohnheiten generell außerhalb des unternehmerischen Handlungsspielraumes lägen.

\section{Win-Win-Situationen herstellen}

Das private Konsumverhalten der Mitarbeiter kann in mehrerer Hinsicht von Bedeutung für Unternehmen sein. Einerseits ist davon auszugehen, dass Mitarbeiter, deren private Verhaltensweisen nachhaltig sind, diese Einstellungen und Werte auch ins Unternehmen tragen und somit zur Entstehung einer Nachhaltigkeitskultur beitragen. Andererseits wirkt innerbetriebliches Nachhaltigkeitsmanagement im Rahmen von CSR vor allem dann überzeugend und ganzheitlich, wenn es durch Maßnahmen ergänzt wird, welche nicht nur organisationalen Zwecken dienen, sondern Ausdruck eines umfassenden Interesses an nachhaltiger Entwicklung sind. Dies kann sich positiv nicht nur auf die Arbeitsatmosphäre und die Arbeitsmotivation auswirken.

Zufriedene Mitarbeiter nehmen auch nach außen eine Multiplikatorfunktion ein, zugunsten des Unternehmensimages und zugunsten der Entstehung einer gesellschaftlichen Nachhaltigkeitskultur. Zudem ist es wahrscheinlich, dass im privaten Konsumbereich der Mitarbeiter nicht nur im Hinblick auf Nachhaltigkeitsziele Probleme existieren, sondern dass auch Mitarbeiter selbst Gestaltungsempfehlungen, Hilfestellungen und Angebote für nachhaltigere Lebensführungsstrategien im Alltag suchen (Schrader 2007). Das kann zu einer WinWin-Situation führen, die es für alle Beteiligten lohnenswert macht, entsprechende Maßnahmen $\mathrm{zu}$ initiieren beziehungsweise an ihnen teilzunehmen.

\section{Nachhaltigen Konsum erfolgreich vorantreiben}

Damit Unternehmen als erfolgreiche Promotoren eines nachhaltigen Konsum- 
verhaltens ihrer Mitarbeiter agieren können und auch die Mitarbeiter dieses zusätzliche Engagement als fruchtbar und hilfreich empfinden, werden im Folgenden verschiedene Determinanten für den Erfolg oder Misserfolg der Maßnahmen herausgestellt.

Zunächst ist zu beachten, dass Mitarbeiter auf ein direktes unternehmerisches Interesse an ihren privaten Lebensweisen gegebenenfalls mit Reaktanz reagieren. Reaktanz tritt immer dann auf, wenn sich der Einzelne in seinem Entscheidungsfreiraum, vor allem in seiner Entscheidungsautorität eingeschränkt fühlt (Brehm 1966). Um Akzeptanz zu generieren sind Maßnahmen auf individuelle Bedarfe abzustimmen. Dieses Vorgehen kann erstens am besten auf Grundlage der Identifikation von Zielgruppen innerhalb der Mitarbeiterschaft gewährleistet werden. Erkenntnisse aus der Zielgruppenkommunikation in der Umwelt- beziehungsweise Nachhaltigkeitsbildung können hierbei zur Anwendung kommen und beispielsweise herausstellen, in welchen Konsumbereichen besonderer Handlungsbedarf besteht und auf welche Weise die Mitarbeiter angesprochen und einbezogen werden sollten (Kleinhückelkotten 2005).

Entsprechend sollten zweitens der Integrationsgrad beziehungsweise die Einbindungsstärke der Mitarbeiter in die Planung und Durchführung der Maßnahmen mit den Bedarfen der Einzelnen korrelieren. Anzunehmen ist, dass eine hohe Mitarbeitereinbindung grundsätzlich Akzeptanz steigernd wirkt, jedoch möglicherweise in manchen Zielgruppen als Überforderung empfunden wird.

Drittens stellt die Einbindung von relevanten Stakeholdern ein weiteres zentrales Kriterium dar. Gewerkschaften und zivilgesellschaftliche Organisationen wie Umweltschutzorganisationen oder Verbraucherverbände können durch ihren Erfahrungsschatz und ihre Expertise die Glaubwürdigkeit erhöhen und das Vertrauen der Mitarbeiter in die Maßnahmen stärken (Freeman 2004). Vor allem aber verringern Stakeholder den gefühlten Unternehmenseinfluss und sorgen somit zusätzlich dafür, dass die Maßnahmen auf Akzeptanz bei den Mitarbeitern stoßen. Die Einbindung der Stakeholder in die Ausgestaltung und Umsetzung der Maßnahmen sollte jedoch nicht zur Annahme verleiten, dass unternehmensspezifische Rahmenbedingungen eine geringe Rolle spielen.

So hängen viertens die Glaubwürdigkeit und der Erfolg von Maßnahmen auch von unternehmerischen Kernkompetenzen und Tätigkeitsfeldern ab. Deshalb ist anzunehmen, dass ein Unternehmen aus Sicht der Mitarbeiter vor allem dann als handlungsberechtigt wahrgenommen wird, wenn die Maßnahmen in einem erkennbaren Zusammenhang zum Geschäftsbereich des Unternehmens stehen. Beispielsweise erscheint ein Elektronikunternehmen vermutlich authentischer, wenn es, als erste Maßnahme, statt biologisch hergestellter Lebensmittel einen effizienten Energieverbrauch unter den Mitarbeitern fördert. Letztlich sind die herausgestellten Determinanten interdependent und sollten in Abstimmung aufeinander in der Planung und Durchführung von Maßnahmen bedacht werden.

\section{Fazit}

Bisher wird die hier dargestellte Promotorenrolle von den Unternehmen kaum offensiv besetzt. Auch die wissenschaftliche Debatte zu dieser Thematik steht noch ganz am Anfang. Das vom interuniversitären Forschungszentrum für Technik, Arbeit und Kultur Graz koordinierte Verbundprojekt „Nachhaltiges Handeln im beruflichen und privaten Alltag" führt im deutschsprachigen Raum erstmalig eine intensive Analyse der Wechselwirkungen zwischen Arbeit und Privatleben im Hinblick auf Nachhaltigkeit durch (ISOE et al. 2008).

Aus unserer Sicht ist das ein erster Schritt zu einer verbesserten Integration der Themenfelder nachhaltiger Konsum, nachhaltiges Management und CSR. Dabei gibt es sowohl für die unternehmerische und gesellschaftliche Praxis, als auch für die Wissenschaft zahlreiche ungenutzte Chancen und offene Fragen. Die- se gilt es im Rahmen zukünftiger Forschungsprogramme und -projekte anzugehen.

\section{Literatur}

Berger, I. E. / Kanetkar, V.: Increasing Environmental Sensitivity Via Workplace Experiences. In: Journal of Public Policy and Marketing. 2/1995, S. 205-215.

Brehm, J. W. / Stires, L. K. / Sensenig, J. / Shaban, J.: The attractivness of an eliminated choice alternative. In: Journal of Experimental Social Psychology 2/1966, S. 301-313.

Freeman, R. E.: The Stakeholder Approach Revisited. In: Zeitschrift für Wirtschafts- und Unternehmensethik (zfwu) 3/2004, S. 228-241.

ISOE, Institut für sozialökologische Forschung/ IFZ, Interuniversitäres Forschungszentrum für Technik, Arbeit und Kultur / IfGP, Institut für Gesundheitsförderung und Prävention / UBZ, Umweltbildungszentrum Steiermark: Nachhaltiges Handeln im beruflichen und privaten Alltag. Internet: http://isoe.info/projekte/ naha.htm (04.05.2009)

Jürgens, K.: Arbeits- und Lebenskraft. Reproduktion als eigensinnige Grenzziehung. Wiesbaden 2006.

Kleinhückelkotten, S.: Suffizienz und Lebensstile. Ansätze für eine milieuorientierte Nachhaltig keitskommunikation. Berlin 2005.

Schrader, U.: The moral responsibility of consumers as citizens. In: International Journal of Innovation and Sustainable Development 1/2007, S. 79-96.

Thøgersen, J.: Spillover processes in the development of a sustainable consumption pattern. In: Journal of Economic Psychology 20/1999, S. $53-81$

I AUTOREN + KONTAKT

Viola Muster ist wissenschaftliche Mitarbeiterin und Ulf Schrader ist Professor für das Fachgebiet Arbeitslehre Wirtschaft/Haushalt der Technischen Universität Berlin.

Technische Universität Berlin, Fachgebiet Arbeitslehre Wirtschaft/Haushalt, Franklinstr. 28/29, 10587 Berlin. Tel.: +493031428769.

E-Mail: Viola.Muster@tu-berlin.de; Schrader@tu-berlin.de 
(c) 20I0 Authors; licensee IÖW and oekom verlag. This is an article distributed under the terms of the Creative Commons Attribution Non-Commercial No Derivates License (http://creativecommons.org/licenses/by-nc-nd/3.o/), which permits unrestricted use, distribution, and reproduction in any medium, provided the original work is properly cited. 